\title{
PESO CORPORAL E ESTADO HÍDRICO DE TRIATLETAS NO IRONMAN BRASIL: UM FATOR DE CORREÇÃO
}

\author{
BODY WEIGHT AND WATER CONDITION IN IRONMAN BRASIL TRIATHLETES: \\ A CORRECTION FACTOR
}

Artigo Original

ORIGINAL ARTICLE

Artículo Original

\author{
PESO CORPORAL Y ESTADO HIIDRICO DE TRIATLETAS EN EL IRONMAN BRASIL: \\ UN FACTOR DE CORRECCIÓN
}

\author{
Roberto Lemos' (Educador Físicol) \\ Francisco Rosa Neto' \\ (Educador ísico) \\ Lourenço Sampaio de Mara' \\ (Médico) \\ Alexandra Amin Lineburger' \\ (Fisioterapeuta) \\ Tales de Carvalho' (Médico) \\ Renata Ramos' (Médica) \\ 1. Universidade do Estado de Santa \\ Catarina, Centro de Educação \\ Física, Fisioterapia e Desportos, \\ Florianópolis, SC, Brasil.
}

\section{Correspondência:}

Rua Ernesto Stodieck, 56, Florianópolis, SC. 88025-130. Brasil. lousmara@hotmail.com

\section{RESUMO}

Introdução: O triatlo Ironman é uma prova de longa duração em que comumente se observam alterações hidroeletrolíticas. A desidratação e hiponatremia são prevalentes e o diagnóstico diferencial entre elas deve levar em conta a variação de peso corporal do atleta. Contudo, deve-se considerar também que as variações são um somatório de fontes hídricas e não hídricas, sendo necessário aplicar um fator de correção para avaliação do real estado hídrico do atleta. Objetivo: Avaliar o estado hídrico do atleta baseado nas variações de peso corporal sem e com aplicação de fator de correção. Método: Vinte e seis atletas foram pesados em três momentos distintos (dois dias antes da prova, imediatamente antes e após a realização). O estado hídrico foi classificado com base no cálculo da variação percentual de peso corporal isolado e com aplicação do fator de correção de $1 \mathrm{~kg}$ proporcional ao atleta de $70 \mathrm{~kg}$. Além disso, foram registrados os principais sinais clínicos e sintomas referidos. Resultados: Nas 48 horas que antecederam a largada houve um ganho médio de peso de $1,2 \mathrm{~kg}$. Após a prova, vinte e três (88,4\%) atletas foram classificados como desidratados inicialmente, porém após a aplicação do fator de correção à variação do peso, esse número caiu para 12 (46,1\%). Dos classificados como desidratação severa houve redução de 7 (26,2\%) para nenhum atleta. Dez atletas $(3,8 \%)$ apresentaram sinais e sintomas de desidratação. Conclusão: A classificação do estado de hidratação baseado nas perdas hídricas durante a prova foi significativamente modificado pela aplicação do fator de correção, sendo sua utilização justificada pelas evidências de que o ganho de peso nas 48 horas anteriores à prova está possivelmente relacionado ao acúmulo muscular de glicogênio e água (fontes não hídricas intravasculares).

Palavras-chave: desidratação, composição corporal, atletas.

\section{ABSTRACT}

Introduction: The Ironman Triathlon is a long-lasting competition where commonly electrolyte changes are observed. Dehydration and hyponatremia are prevalent and the differential diagnosis between them should take into account the body weight variation of the athlete. Nevertheless, one should also consider that variations are a summation of water and no-water sources, being necessary to apply a correction factor to evaluate the real water condition of the athlete. Objective: To evaluate the water condition of the athlete based on body weight changes with and without correction factor applied. Method: Twenty-six athletes were weighed in three different times (two days before the competition, immediately before and after performance). The water was classified by calculating the percentage of isolated body weight variation and application of correction factor of $1 \mathrm{~kg}$ proportional to the athlete of $70 \mathrm{~kg}$. In addition, the main clinical signs and symptoms were recorded. Results: In the 48 hours before the start there was an average weight gain of $1.2 \mathrm{~kg}$. After the race, 23 (88.4\%) athletes were classified as dehydrated initially but after applying the correction factor to the weight change, this number dropped to 12 (46.1\%). Those classified as severe dehydration decreased by 7 (26.2\%) to no athlete. Ten athletes (3.8\%) presented signs and symptoms of dehydration. Conclusion: The classification of hydration status based on water loss during the race was significantly modified by the application of the correction factor, and its use is justified by evidence that weight gain within 48 hours prior to the race is possibly related to the muscle glycogen and water accumulation (no intravascular water sources).

Keywords: dehydration, body composition, athletes.

\section{RESUMEN}

Introducción: El triatlón Ironman es una prueba de larga duración en la que se observan comúnmente alteraciones hidroelectrolíticas. La deshidratación e hiponatremia son prevalentes y el diagnóstico diferencial entre éstas debe llevar en cuenta la variación de peso corporal del atleta. Sin embargo, se debe considerar también que las variaciones son una sumatoria de fuentes hídricas y no hídricas siendo necesario aplicar un factor de corrección para evaluación del real estado hídrico del atleta. Objetivo: Evaluar el estado hidrico del atleta con base en las variaciones de peso corporal sin y con aplicación de factor de corrección. Método: Veintiséis atletas fueron pesados en tres momentos distintos (dos días antes de la prueba, inmediatamente antes y después de la realización de la misma). El estado hídrico fue clasificado con base en el cálculo de la variación porcentual de peso corporal aislado 
y con aplicación del factor de corrección de $1 \mathrm{~kg}$ proporcional al atleta de $70 \mathrm{~kg}$. Además, fueron registradas las principales señales clínicas y sintomas referidos. Resultados: En las 48 horas que antecedieron a la largada hubo un aumento medio de peso de 1,2 kg. Después de la prueba, veintitrés (88,4\%) atletas fueron clasificados como deshidratados inicialmente, aunque después de la aplicación del factor de corrección a la variación de peso, ese número cayó para $12(46,1 \%)$. Y de los clasificados como deshidratación severa hubo reducción de 7 (26,2\%) para ningún atleta. Diez atletas (3,8\%) presentaron señales y síntomas de deshidratación. Conclusión: La clasificación del estado de hidratación con base en las pérdidas hídricas durante la prueba fue significativamente modificada por la aplicación del factor de corrección, siendo su uso justificado por las evidencias de que el aumento de peso en las 48 horas antes de la prueba está posiblemente relacionado a la acumulación muscular de glicógeno y agua (fuentes no hídricas intravasculares).

Palabras clave: deshidratación, composición corporal, atletas.

\section{INTRODUÇÃO}

O triathlon ironman é uma prova de longa duração que consiste em 3,8 km de natação, 180 km de ciclismo, seguidos de 42,2 km de corrida, com duração média de treze horas ${ }^{1}$ e tempo limite de 17 horas.

Em eventos como este, são comuns diversas alterações orgânicas ${ }^{2,3}$, dentre elas a desidratação e hiponatremia que têm sinais e sintomas semelhantes ${ }^{3,4}$, sendo de suma importância o diagnóstico preciso uma vez que os tratamentos são distintos para cada entidade. A desidratação ocorre devido à exposição prolongada a fatores ambientais, como o calor e a umidade que dificultam a manutenção do equilíbrio térmico, causando perda hídrica de 1 a $2 \mathrm{~L} / \mathrm{h}$ pela sudorese $\mathrm{e}^{2,4}$. Por outro lado, a hiponatremia nesta população está relacionada principalmente à ingesta hídrica em excesso ao longo da prova'.

É sugerido que além dos sinais e sintomas, a avaliação da variação de peso corporal antes e após a prova de triatlo Ironman seja um parâmetro útil no diagnóstico diferencial entre desidratação (perda de peso) e hiponatremia (ganho de peso) uma vez que reflete o estado hídrico do atleta ${ }^{5}$, sendo considerado um marcador simples ${ }^{5}$, prático e de boa acurácia na determinação de alterações agudas no estado hídrico do atleta ${ }^{6}$.

O estado hídrico pode ser mensurado pela variação entre o valor do peso antes e após a atividade física. No entanto é importante observar que a perda hídrica de um atleta em atividade de longa duração é um somatório de fontes hídricas e não hídricas proveniente da oxidação da gordura, carboidratos e água contida no interior do glicogênio ${ }^{7-12}$. Rogers et al. ${ }^{7}$ sugerem que a perda de 1 a $2 \mathrm{~kg}$ de outras fontes diferentes da perda hídrica plasmática é responsável pela variação de peso.

Hipotetisamos que a avaliação do estado hídrico baseada na variação percentual do peso corporal apresentada por triatletas de ironman não reflete a sua real condição hídrica. Desta forma o objetivo do estudo é avaliar o estado hídrico do atleta baseado nas variações de peso corporal sem e com aplicação de um fator de correção relacionando com e sinais e sintomas clínicos.

\section{MATERIAIS E MÉTODOS}

A amostra foi selecionada de forma intencional e composta por 26 atletas amadores integrantes de uma equipe de triatlo de Florianópolis, SC, Brasil, sendo dois do gênero feminino e 24 do gênero masculino. Estes foram devidamente informados sobre os procedimentos de pesquisa e assinaram o Termo de Consentimento Livre e Esclarecido aceitando participar do estudo, aprovado previamente pelo Comitê de Ética em Pesquisa da Universidade do Estado de Santa Catarina (CEFID), Florianópolis, SC, Brasil, sob o protocolo 16/05.

Para verificação da massa corpórea, foi utilizada uma balança eletrônica da marca Plenna (modelo Lithium Digital Sport, com precisão de 100 gramas- Brasil) posicionada em uma superfície dura e plana. Os atletas foram pesados descalços, usando trajes de banho, em três momentos distintos: dois dias antes, 1 hora antes e logo após o termino da prova. Os dados foram representados em kilogramas (Kg) com uma casa decimal.

A variação de peso corporal foi expresso em percentual de perda ou ganho de peso utilizando-se a seguinte fórmula: (Ppré- Ppós)x 100/ Ppré, sendo Ppré $=$ Peso pré-prova. Ppós = Peso pós-prova.

A determinação da variação de peso corporal utilizando-se um fator de correção se fez através da fórmula: (Ppré-FC)-Ppós x 100/(Ppré-FC), sendo Ppré=peso pré-prova. Ppós=peso pós-prova. Fc=Fator de correção. Fator de correção=1 Kg.

Foram observados e registrados por profissional médico competente, sinais referentes à frequência cardíaca em posição ortostática pela medida do pulso radial em um minuto, pressão arterial sistólica e diastólica por esfigmanômetro de mercúrio, turgor da pele e mucosas oculares e sintomas quanto à sensação de sede.

\section{RESULTADOS}

A amostra estudada constitui-se de 26 atletas, dos quais dois eram do gênero feminino e 24 do gênero masculino. A idade média foi de 37,1 \pm 7,9 anos, com idade mínima de 21 anos e máxima de 51 anos. O tempo médio de prova dos participantes do estudo foi de $12 \mathrm{~h}^{\prime} \mathrm{O}^{\prime} \pm$ 1h30', com menor tempo de 9h36' e o maior de 16h36'.

As médias de massa corporal dos atletas dois dias que antecederam, imediatamente antes, imediatamente após e a variação dentro de cada momento são apresentadas na tabela 1.

Tabela 1. Média de peso dos atletas de entre 2 dias e pré-competição.

\begin{tabular}{c|c|c|c|c|c|c|c}
\hline Variável & $\mathbf{n}$ & $\begin{array}{c}\text { Ppré } \\
\mathbf{2} \text { dias }\end{array}$ & Ppré & Ppré-fc & Ppós & $\begin{array}{c}\boldsymbol{\Delta} \\
\mathbf{( k g )}\end{array}$ & $\begin{array}{c}\boldsymbol{\Delta} \\
\mathbf{( \% )}\end{array}$ \\
\hline Massa corporal (Kg) & 26 & $69,4(7,3)$ & $70,6(7,4)^{*}$ & & & 1,2 & 1,7 \\
\hline Massa corporal (Kg) & 26 & & $70,6(7,4)$ & & $67,6(6,9)^{*}$ & $-3,0$ & $-4,2$ \\
\hline Massa corporal (Kg) & 26 & & & $69,6(6,9)$ & $67,6(6,9)^{*}$ & $-2,0$ & $-2,8$ \\
\hline
\end{tabular}

Ppré2 dias: peso dois dias antes da prova; Ppré: peso imediatamente antes da prova; Ppós: peso imediatamente após a prova; Ppré-fc: Peso pré prova subtraído fator de correçăo; $\Delta$ : variação entre os pesos. DP: desvio padrão; Valores são média ( desvio padrão); *Valor de $p<0,01$.

As variações de peso percentuais sem e com aplicação do fator de correção são apresentadas de forma estratificada na tabela 2, caracterizando a incidência do grau de desidratação ou hiper-hidratação dos atletas imediatamente após a prova.

Os sinais observados e sintomas referidos pelos atletas por ocasião da chegada são apresentados na tabela 3. 
Tabela 2. Distribuição dos atletas por grau de desidratação ou hiperhidratação.

\begin{tabular}{c|c|c|c|c|c}
\hline & $\begin{array}{c}\text { Manutenção } \\
\text { ou ganho de } \\
\text { peso }\end{array}$ & $\begin{array}{c}\text { Desidrata- } \\
\text { ção leve }\end{array}$ & $\begin{array}{c}\text { Desidratação } \\
\text { moderada }\end{array}$ & $\begin{array}{c}\text { Desidratação } \\
\text { severa }\end{array}$ & Total \\
\hline $\begin{array}{c}\text { Sem fator de } \\
\text { correção }\end{array}$ & $1(3,8 \%)$ & $3(11,5 \%)$ & $16(61,3 \%)$ & $7(26,8 \%)$ & 26 \\
\hline $\begin{array}{c}\text { Com o fator } \\
\text { de correção }\end{array}$ & $3(11,5 \%)$ & $11(42,2 \%)$ & $12(46 \%)$ & - & 26 \\
\hline
\end{tabular}

Tabela 3. Sinais e sintomas referidos pelos atletas imediatamente após prova.

\begin{tabular}{c|c}
\hline Sintoma & $\begin{array}{c}\mathbf{N}^{\circ} \text { de relatos } \\
\text { (\% dos participantes) }\end{array}$ \\
\hline Sensação de sede & $10(38 \%)$ \\
\hline Sinais & $9(34 \%)$ \\
\hline $\begin{array}{c}\text { Pressão arterial sistólica }<100 \text { ou } \\
\text { diastólica }<60 \mathrm{mmHg}\end{array}$ & $7(26 \%)$ \\
\hline Frequência cardíaca $>100 \mathrm{bpm}$ & $7(26 \%)$ \\
\hline Mucosas secas & $9(34 \%)$ \\
\hline Turgor de pele diminuído &
\end{tabular}

\section{DISCUSSÃO}

O estudo realizado confirma a hipótese que a avaliação do estado hídrico baseada na variação percentual do peso corporal apresentada por triatletas de Ironman não reflete a sua real condição hídrica imediatamente após a prova, sendo necessária a utilização de um fator de correção na análise desta variação. Determinar o estado hídrico de forma fidedigna mostrou-se um grande desafio, uma vez que essa determinação baseia-se nas mudanças do peso da massa corporal, sendo que $500 \mathrm{mg}$ corresponde a 480-500 $\mathrm{ml}^{13}$. Porém, o grau de desidratação é superestimado quando calculado unicamente pela diferença de peso corporal determinado pré e pós prova, pois há que se considerar as alterações de peso corporal de um atleta de triatlo Ironman provenientes de fontes não hídricas ${ }^{7,9}$.

A análise de dados preliminares do Ironman de 2002 indicou que o grau de desidratação encontrado não correspondia à condição clínica dos atletas ${ }^{14}$. Foi, então, considerada a necessidade de se aplicar um fator de correção,para se avaliar corretamente a perda hídrica e, consequentemente, o grau de desidratação de um atleta, por meio das alterações de peso corporal. Dessa forma, aplicou-se o fator de correção de $1 \mathrm{~kg}$ a um atleta padrão de $70 \mathrm{~kg}$ e proporcionalmente aos atletas de maior e menor peso. Acredita-se, então, que a avaliação do grau de desidratação se aproximou mais do real, permitindo uma avaliação mais fidedigna. Rogers et al. ${ }^{7}$ sugerem que a perda de 1 a $2 \mathrm{~kg}$ de outras fontes diferentes da perda hídrica plasmática são responsáveis pela perda de peso?

\section{REFERÊNCIAS}

1. Mara LS, Lemos R, Brochi L, Rohlfs MPCI, Carvalho T. Alterações hidroeletrolíticas agudas ocorridas no Triatlon Ironman Brasil. Rev Bras Med Esporte. 2007;13(6):397-401.

2. Noakes TD, Goodwin N, Rayner BL, Branken T, Taylor RK. Water intoxication: a possible complication during endurance exercise, 1985. Wilderness Environ Med. 2005;16(4):221-7.

3. Burger-Mendonca M. Comportamento do íon magnésio em prova triathlon meio-ironman. Motricidade. 2008;4(2):55-60.

4. Sawka MN, Montain SJ, Latzka WA. Hydration effects on thermoregulation and performance in the heat. Comp Biochem Physiol A Mol Integr Physiol. 2001;128(4):679-90. Review.

5. Casa DJ, Armstrong LE, Hillman SK, Montain SJ, Reiff RV, Rich BS, et al. National athletic trainers' association position statement: fluid replacement for athletes. J Athl Train. 2000;35(2):212-24

6. Cheuvront SN, Carter R 3rd, Montain SJ, Sawka MN. Daily body mass variability and stability in active men undergoing exercise-heat stress. Int J Sport Nutr Exerc Metab. 2004;14(5):532-40.

7. Rogers G, Goodman C, Rosen C. Water budget during ultra-endurance exercise. Med Sci Sports Exerc. 1997;29(11):1477-81

8. Speedy DB, Faris JG, Hamlin M, Gallagher PG, Campbell RG. Hyponatremia and weight changes in an ultradistance triathlon. Clin J Sport Med. 1997;7(3):180-4.
Estudos prévios sugerem que na semana anterior a prova há variação de ganho peso devido ao acúmulo de glicogênio e água no tecido muscular ${ }^{15}$. Nosso estudo verificou que ocorreu ganho de peso de 1,2 kg (tabela 1) nas 48 h anteriores à largada em concordância com os achados de Sharwood et al. ${ }^{16}$ que relatou um ganho de 0,9 kg. Em controvérsia, Speedy et al. ${ }^{8}$ não verificaram diferenças de peso dois dias antes e imediatamente antes da prova sugerindo que a primeira pesagem poderia ser utilizada como referência de peso pré-prova devido a facilidade para avaliar os atletas.

A fim de classificar o estado hídrico do atleta, considerou-se que a perda percentual de 3\% da massa corporal denota uma desidratação leve e o atleta pode experimentar diminuição de desempenho, sem comprometimento clínico. A perda entre 3 e 6\% corresponde a uma desidratação moderada com prejuízo da termorregulação, aparecimento de cãibras, contraturas e colapso. A perda acima de $6 \%$ da massa corporal corresponde à desidratação severa e o atleta pode apresentar convulsões, coma e até óbito. A perda de 1 kg ou manutenção e ganho de peso representa respectivamente um estado de euhidratação ou superhidratação ${ }^{15}$.

Ao avaliarmos a condição clínica dos atletas verificamos que 38\% destes apresentavam sinais e sintomas sugestivos de desidratação (tabela 3), que não foi concordante com o alto percentual (88\%) de classificados como desidratação moderada e/ou severa (tabela 2) quando não consideramos o fator de correção nesta avaliação. Porém ao corrigirmos o peso do atleta no momento imediatamente antes da prova subtraindo deste $1 \mathrm{Kg}$ referente a um atleta de $70 \mathrm{~kg}$ e de forma proporcional aos demais, verificou-se consistência entre o percentual de atletas desidratados clinicamente (38\%) e entre os avaliados pela variação de peso corporal corrigida (46\%). Desta forma, a utilização do fator de correção utilizado se mostrou adequado, somado ao fato da variação de peso absoluto encontrado em nosso estudo entre os momentos dois dias e imediatamente antes da prova ter sido muito próximo ao fator de correção utilizado, sugerindo que perdas de até 1 kg em média não representam um estado de alteração hídrica.

\section{CONCLUSÃO}

O presente estudo aponta que ao avaliarmos o estado hídrico de atletas participantes do triatlo Ironman baseado nas variações de peso corporal, deve-se utilizar um fator de correção para melhor refletir sua real condição.

Todos os autores declararam não haver qualquer potencial conflito de interesses referente a este artigo.

9. Speedy DB, Noakes TD, Rogers IR, Thompson JM, Campbell RG, Kuttner JA, et al. Hyponatremia in ultradistance triathletes. Med Sci Sports Exerc. 1999;31(6):809-15.

10. O'Toole ML, Douglas PS, Laird RH, Hiller DB. Fluid and electrolyte status in athletes receiving medical care at an ultradistance triathlon. Clin J Sport Med. 1995;5(2):116-22.

11. Noakes TD. Fluid replacement during exercise. Exerc Sport Sci Rev. 1993;21:297-330.

12. Speedy DB, Campbell R, Mulligan G, Robinson DJ, Walker C, Gallagher P, et al. Weight changes and serum sodium concentrations after an ultradistance multisport triathlon. Clin J Sport Med. 1997;7(2):100-3.

13. Guyton TAGA. Circulatory physiologyii: dinamics and control of the body fluids. Philadelphia: Saunders; 1975

14. Mara LS, Zunino Jn, Carminatti L, Dacar M, Della Giustina M, et al. Análise crítica de estudo sobre alteraçōes orgânicas agudas observadas em participantes de evento de ultra endurance. Rev Bras Med Esporte. 2003;9(Suppl 1):54.

15. Speedy DB, Rogers IR, Noakes TD, Thompson JM, Guirey J, Safih S, et al. Diagnosis and prevention of hyponatremia at an ultradistance triathlon. Clin J Sport Med. 2000;10(1):52-8.

16. Sharwood K, Collins M, Goedecke J, Wilson G, Noakes T. Weight changes, sodium levels, and performance in the South African Ironman Triathlon. Clin J Sport Med. 2002;12(6):391-9. 